Chirurg 2010 $81: 321-322$

DOI 10.1007/s00104-010-1897-3

Online publiziert: 11. März 2010

๑) Springer-Verlag 2010

\author{
J.R. Siewert \\ Universitätsklinikum Heidelberg
}

\section{Klinische Forschung in der Chirurgie}

\section{LOM als Anreiz - oder als Fehlsteuerung?}

Die Sicherung des medizinischen Fortschritts gehört neben der Lehre zu den gesetzlichen Vorgaben der Hochschulmedizin. Der Erfolg in Forschung und Lehre ist somit auch entscheidendes Qualifikationsmerkmal für Universitätsklinika und damit auch für die verschiedenen klinischen Fachgebiete. Da insbesondere für die Chirurgie Organisation und Qualitätssicherung der klinischen Forschung außer man kooperierte mit einem theoretischen Institut, das sich der Grundlagenforschung widmet - schwierig und herausfordernd ist, erscheint eine Bestandsaufnahme aus chirurgischer Sicht zum Thema „Klinische Forschung“ sinnvoll und wichtig.

Wie unterschiedlich dabei die Beurteilung der Stellung der deutschen chirurgischen Forschung im internationalen Vergleich ausfallen kann, können Sie diesem Heft entnehmen. Nicht zuletzt war es unter diesem Gesichtspunkt schon immer wichtig, einen Standard für die Bewertung der klinischen Forschung einzuführen und diesen zur Grundlage der $\mathrm{Zu}$ teilung von Landesmitteln für Forschung und Lehre zu machen.

Schon im Jahre 1995 hat der Wissenschaftsrat erstmals gefordert, dass die Landeszuschüsse für Forschung und Lehre nach wissenschaftlichen Leistungskriterien vergeben werden müssen. Man sah darin eine Grundvoraussetzung dafür, dass an den medizinischen Fakultäten durch Förderung von Exzellenz eine verbesserte internationale Wettbewerbsfähigkeit erreicht werden könnte. Im Jahre 2007 hat der Wissenschaftsrat in sei- ner Denkschrift „Allgemeine Empfehlung für die Universitätsmedizin“ befriedigt festgestellt, dass die leistungsorientierte Mittelverteilungsverfahren (LOM) auf Länderebene und in den Hochschulen selbst in den vergangenen 12 Jahren nahezu flächendeckend umgesetzt sei. Durch die LOM ist ein wettbewerblicher Einheitsrahmen geschaffen sowie Transparenz hinsichtlich der Budgetierung einerseits und der Leistung andererseits erreicht worden.

Für die tägliche Diskussion muss zwischen innerer und äußerer LOM unterschieden werden. Im Rahmen der äußeren LOM werden Universitätsklinika untereinander bewertet und in einen unterschiedlich großen Anteil Landesmittel umverteilt. Die innere LOM betrifft dagegen das Innenverhältnis der verschiedenen Fachgebiete in einer Fakultät; auch hier ist das Ziel letztendlich die Umverteilung von Landesmitteln.

Zwei semantische Probleme liegen auf der Hand:

- Zum einen bedeutet eine leistungs-

orientierte Vergabe der Mittel für

Forschung und Lehre zwangsläu-

fig immer auch eine Umverteilung

von vielen auf wenige. Dabei be-

trifft die Konkurrenz nicht nur Ab-

teilungen und Kliniken innerhalb einer Fakultät, sondern auch die medizinischen Fakultäten selbst innerhalb eines Bundeslandes. Umverteilen ist immer schmerzhaft, weil es nicht nur wenige Gewinner, sondern meistens auch viele Verlierer gibt, die vielleicht in den universitären Gremien meinungsbildend sein können.

- Zum anderen wurde die LOM von Anfang an durch die unabdingbare Voraussetzung der Trennungsrechnung belastet. Allein der Begriff „Trennungsrechnung“ war und ist negativ besetzt. Erst spät hat man versucht diesen Begriff durch den Begriff „Transparenzrechnung“ zu ersetzen. Mittelflüsse müssen separiert werden, wohlwissend, dass ein nicht auflösbarer Graubereich verbleibt. Darüber hinaus bedingt diese Prozedur einen hohen Personalaufwand.

Rasch wurde klar, dass man die LOM offenbar von wenigsten zwei Seiten sehen muss:

- Einerseits unter den mehr akademischen Gesichtspunkten:Wie erfasst man wissenschaftliche Leistung? Wie kann man sie messen bzw. kann man sie überhaupt quantifizieren? Hierher gehört die Diskussion um den Impact- und H-Faktor. Wie gewichtet man Drittmittel?

- Andererseits die mehr praktische Sicht:Wie geht man mit der LOM im Alltag von Fakultät und Universitätsklinikum um? Akzeptiert man die daraus resultierenden Steuerungselemente und sind diese höherrangig anzusiedeln als andere traditionelle Steuerungselemente?

Mit anderen Worten: Es gilt die Frage zu beantworten: 
- Regiert LOM die Hochschulmedizin? Oder:

- Versteht die Hochschulmedizin die LOM lediglich als Mittel zum Zweck und damit als nur einen Aspekt in der Steuerung der Hochschulmedizin?

Von Anfang an war klar, dass die Entwicklung von Kriterien für eine leistungsorientierte Zuteilung als Prozess bzw. als lernendes System verstanden werden musste. Inzwischen sind die Kriterien der LOM in den verschiedenen Bundesländern mit vielen nicht direkt leistungsbezogenen Kriterien, wie z. B. Promotions- und Habilitationsquote, Frauenquote etc., durchmischt worden. Man kann leicht den Eindruck gewinnen, dass die LOM sich zunehmend mehr in die Richtung auf ein neues Steuerungsinstrument der Wissenschaftsministerien entwickelt und den primären Sinn der internationalen Wettbewerbsfähigkeit aus den Augen verliert.

Man erinnere sich: Ursprünglich war die LOM ausschließlich auf die Parameter Qualität und Zahl erfolgter Publikation und die Höhe und Herkunft eingeworbener Drittmittel fokussiert. Erst sekundär kam die Lehre als Parameter hinzu. Allein diese drei Parameter geben schon genug Anlass zu Diskussionen hinsichtlich der Bewertungskriterien.

Auch in der jetzt aktuellen Diskussion um die Kooperation zwischen außeruniversitärer und universitärer Forschung begegnet uns dieses Problem wieder. Sind Bundesmittel LOM relevant? Oder gilt die unsinnige Trennung von Bundesmitteln und Landesmitteln als Konsequenz der Föderalismusreform I auch hier?

Alles in allem offenbar doch noch ein Thema von hoher Aktualität! Die Diskussion dieser Probleme ist uns deswegen ein besonderes Anliegen - sowohl der medizinischen Fakultäten, die ja unmittelbar Betroffene sind, aber auch der Universitätsklinika, die mehr in die Problematik der praktischen Umsetzung involviert sind, und der einzelnen Fächer wie z. B. die Chirurgie. Die Chirurgie lebt aber auf keiner Insel, sondern in der Gemeinschaft der Fächer, z. B. in einer Fakultät. Sie wird weder Sonderrechte noch Mitleid beanspruchen können. Vielmehr ist klinische Forschung mehr denn je interdisziplinär. Ja sie entsteht und entwi- ckelt sich bevorzugt an den Fächergrenzen; sie bedarf deshalb auch interdisziplinärer Richtlinien und Parameter, die für alle Beteiligten gleich sind.

Nur Mut - auch chirurgische Forschung kann sich messen lassen und sich dem interdisziplinären Vergleich stellen.

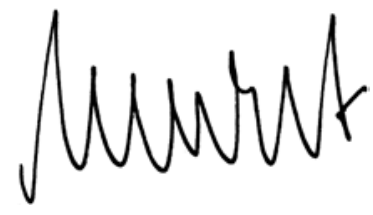

Prof. Dr. Dr. h.c. Siewert

\section{Korrespondenzadresse}

Prof. Dr. Dr. h.c. J.R. Siewert

Universitätsklinikum Heidelberg Im Neuenheimer Feld 672, 69120 Heidelberg

JR.Siewert@med.uni-heidelberg.de

\section{Deutsch als Wissenschaftssprache}

Der Deutsche Akademische Austauschdienst (DAAD) hat ein Memorandum zur Förderung von Deutsch als Wissenschaftssprache vorgelegt. Darin fordert er, dass die deutsche Sprache in der zunehmend vom Englischen dominierten internationalen Wissenschaft ihre traditionsreiche Stellung bewahren muss.

Zu den Kernaufgaben des DAAD gehört die Förderung von Deutsch als Fremdsprache ebenso wie die Internationalisierung der deutschen Hochschulen. Während auf der einen Seite die weltweite Kommunikationsfähigkeit der Forschung gewährleistet sein muss, soll auf der anderen Seite deutschen Wissenschaftlern die Möglichkeit erhalten bleiben, ihre Erkenntnisse in der eigenen Muttersprache und der entsprechenden sprachlichen Nuancierung zu erzielen und zu vermitteln.

Zudem sollen die Vorteile des Erwerbs der deutschen Sprache - wie der Zugang zu einem der bedeutendsten Standorte für Wissenschaft, Wirtschaft und Kultur - stärker hervorgehoben werden. Die beste Werbung für Deutsch als Wissenschaftssprache, so lautet eine Leitlinie des Memorandums, ist die wissenschaftliche Exzellenz deutscher Hochschulen. In englischsprachigen Studiengängen soll verstärkt auf Angebote hingewirkt werden, die ein Erlernen der deutschen Sprache ermöglichen, um die soziale Integration der ausländischen Gäste an den Hochschulen zu gewährleisten.

Deutscher Akademischer Austauschdienst e.V. (DAAD), www.daad.de 